\title{
Visualization research on the influence of an ultrasonic degassing system on the operation of a hydraulic gear pump
}

\author{
Piotr Antoniak ${ }^{1,1}$, Jarosław Stryczek ${ }^{1}$, Michat Banaś ${ }^{1}$, Oleksandr Lyhovskyi ${ }^{2}$, Ihor \\ Gryshko $^{2}$, Andrii Zilinskyi ${ }^{2}$, and Vasyl Kovalov ${ }^{2}$ \\ ${ }^{1}$ Wrocław University of Science and Technology, Faculty of Mechanical Engineering, Poland \\ ${ }^{2}$ National Technical University of Ukraine "Igor Sikorsky Kyiv Polytechnic Institute", Ukraine
}

\begin{abstract}
Gear pumps make a group of the most popular hydraulic energy generators. Research and development works concerning those units have been going on for decades, and thanks to them gear pumps feature very good operating parameters. However, even well-designed gear pumps will not work properly if the physical properties of the working fluid are incorrect. One of such properties is compressibility of the fluid, which largely depends on the amount of gas dissolved in the medium. For this reason, the aim is to reduce the amount of gas dissolved in the working medium. It can be done using both chemical and physical methods. Because chemical methods can affect the chemical composition of the working fluid, it is the physical methods that are usually used in hydraulic systems.

This paper presents preliminary visualization research into the influence of an ultrasonic degassing system on the operation of a hydraulic gear pump. Apart from that, operation of such a system and its theoretical impact on the work of the gear pump is discussed Experimental study, using a high-speed camera, was carried out in order to verify the theoretical description.
\end{abstract}

\section{Introduction}

High dynamic characteristics, reliability and durability of hydraulic drive systems are connected with observation of working fluids' service properties. One way to stabilize the fluid properties is to reduce the gas content.

There are chemical and physical degassing methods. One of the physical methods is ultrasonic degassing which consists in injecting of high intensity ultrasonic oscillations into the fluid. If the intensity of the ultrasonic oscillations is lower than the limit of cavitation emergence, the unsolved air bubbles begin to oscillate in time. Due to that, a bubble the size of which is smaller than the resonant size, pulsates in phase with pressure oscillations and moves according to wave pressure changes. Contrary to that, a bubble that is of the size larger than the resonant size, moves to pressure nodes of a standing ultrasonic wave [1-3].

\section{Corresponding author: piotr.antoniak@pwr.edu.pl}


The resonant bubble size is the bubble radius whose natural oscillations frequency is equal to the forced oscillations frequency of an ultrasonic deformation wave. During the process, each individual bubble moves at the velocity defined by formula (1)

$$
v=\frac{R_{0}^{2}}{10 \mu\left(p_{0}+\frac{4 \sigma}{3 R_{0}}\right)} p_{a} \frac{\partial p_{a}}{\partial x}
$$

where: $\sigma$ - surface tension coefficient; $R_{0}$ - initial bubble radius; $p_{0}$ - hydrostatic pressure; $\mu$-dynamic fluid viscosity coefficient; $p_{a}$ - sound pressure generated by sound field; $x$ - spatial coordinate.

As a result, bubbles are accumulated in the nodal regions of the pressure standing wave [4], coalesce (stick together) and emerge onto the fluid surface. However, it should be said that efficiency of the diffusion based degassing process is practically negligible.

On the other hand, if the intensity of an ultrasonic wave in the expansion phase is high enough to drop the fluid pressure below the saturation vapour pressure, the ultrasonic cavitation appears. For such condition, the cavitation bubbles are created from cavitation embryos wherein the embryos which can be excited by the ultrasonic cavitation must feature a radius no smaller than the critical radius defined by formula (2) [2,3]

$$
R_{c r}=\sqrt{\frac{3 R_{0}^{3}}{2 \sigma}\left(p_{0}+\frac{2 \sigma}{R_{0}}\right)}
$$

In the expansion phase, while increasing the size of the bubble and by the decrease in gas concentration inside that bubble, the gas dissolved in the fluid diffuses into the bubble. At the same time, the bubble size grows due to fluid evaporation from the bubble inner surface. This process leads to the cooling of the bubble's surface, as well as the vapour-gas mixture in its interior. The resulting temperature drop additionally supports the evaporation process. Similarly, during the compression phase, the bubble size decreases and the vapourgas diffusion from the bubble to the fluid appears. Because the amount of the diffused gas is proportional to the bubble surface, which is greater during the expansion phase, the growth of the gas mass and the bubble size takes place.

If the oscillation amplitude is high enough, the cavitation bubble does not have time to collapse in the compression phase. Instead of this, the bubble size is slightly reduced. Consequently, the cavitation bubble oscillates, accumulating energy and the amount of gas entrapped in its interior up to the drop point. At that moment, depending on its proximity to adjacent bubbles and to solid surfaces, spherical shock waves or energy-intensive cumulative jets may appear. This, usually harmful, process additionally increases the efficiency of the degassing process. In contrary to the above, if the cavitation bubble bursts during the compression phase, it becomes a cavitation embryo again. That process is accompanied by intense micro-flows, which accelerate the movement, collision and coalescence of the cavitation bubbles.

The above analysis shows that the most effective and expedient method is the use of a high-amplitude ultrasonic emitter with a well-developed radiation surface. Such a device guarantees the occurrence of cavitation bubbles oscillation and micro-flows which intensify the degassing process. As shown in Fig. 1 such a system is highly efficient. The percentage amount of gas estimated by the volumetric method indicates unquestionably that compared 
to other methods, the ultrasonic degassing system is much faster, more effective and cases deeper decreasing of the dissolved and the undissolved gas concentration in a fluid.

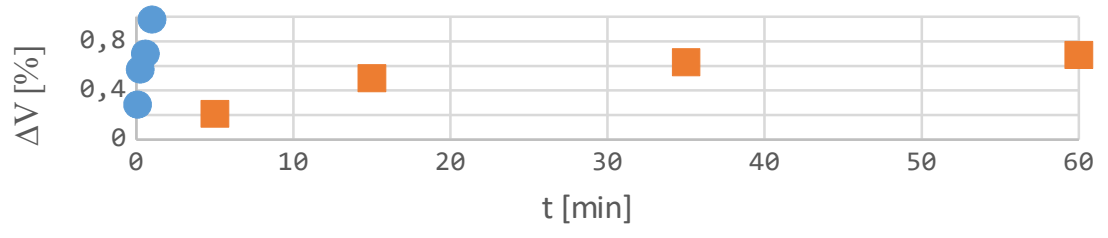

Fig. 1. Comparison of efficiency of ultrasonic (blue circles) and sedimentation (red squares) degassing system used for degassing of Envirologic 3046 oil.

\section{Test stand}

The diagram of a test stand has been shown in Fig. 2a, and its view in Fig. $2 b$.
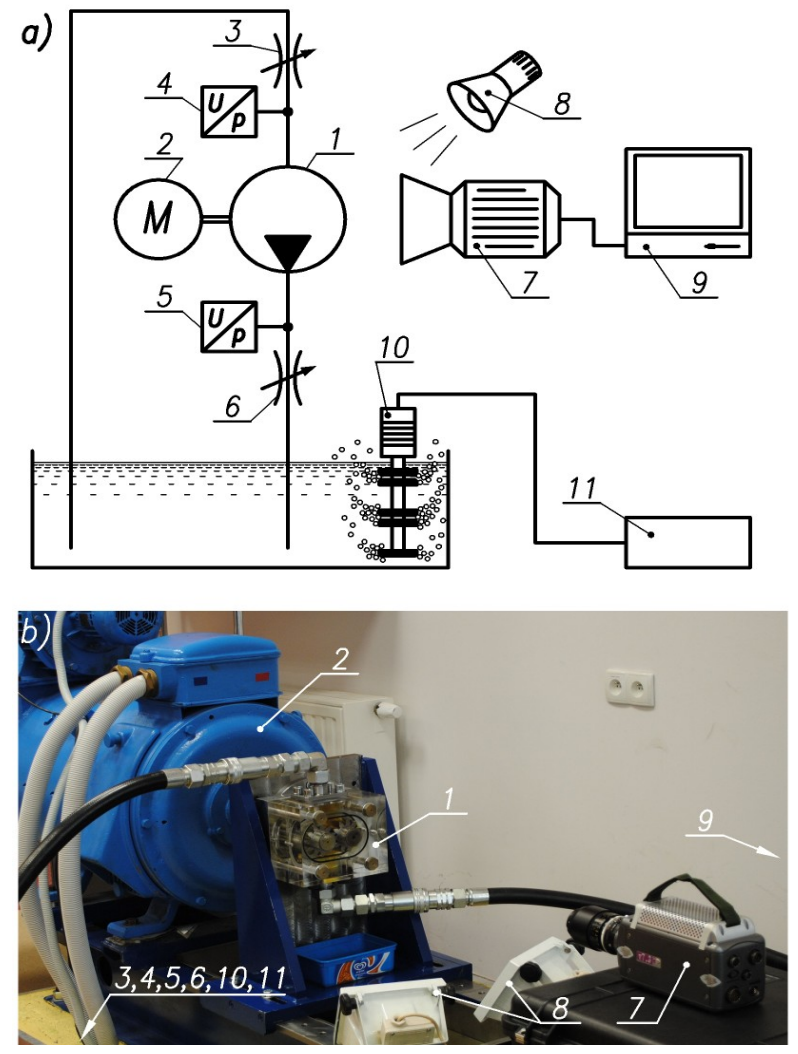

Fig. 2. Test stand

a) simplified diagram, b) view of the test stand

The test stand consists of a hydraulic-mechanical system, a degassing system and a visualization system. The mechanical system consists of an experimental gear pump (1) and a $50 \mathrm{~kW}$ DC electric motor (2) whose speed is adjusted by a controller with a built-in speed indicator. The pressure of the working fluid, a typical ISO VG 68 hydraulic oil, at the inlet of the pump is controlled by a throttle valve (3) and the WIKA A-10/-1...+15 bar pressure transducer (4). Similarly, the pump output pressure is set using a throttle valve (6) and the WIKA A-10/0...+100 bar pressure transducer (5). 
The degassing system consists of an ultrasonic emitter (10) mounted inside of a 60 liter hydraulic tank. The emitter is powered by a custom designed, high power electronic driver (11). The actual power used by the ultrasonic emitter is adjusted by a knob built in the driver.

Last but not least, there is a visualization system, the function of which is to record the phenomena and flow processes occurring in the pump. The system consists of a high-speed camera (7) (Phantom v 7.3 by Visual Research), and a computer (9) with dedicated software. The software, Phantom Camera Control Software, also by Vision Research, has been used for the analysing and processing of the recorded images. The system is completed with a light source (8) consisting of two 500 watt general purpose halogen lamps.

The test stand enables conducting research on the influence of the degassing system on the flow phenomena and processes in a hydraulic gear pump. A similar system had been used repeatedly for visualization research by a team of researchers from Wrocław University of Science and Technology [5-10], and it proved to be sufficient.

\subsection{Experimental pump}

Fig. 3 shows the experimental external gear pump.
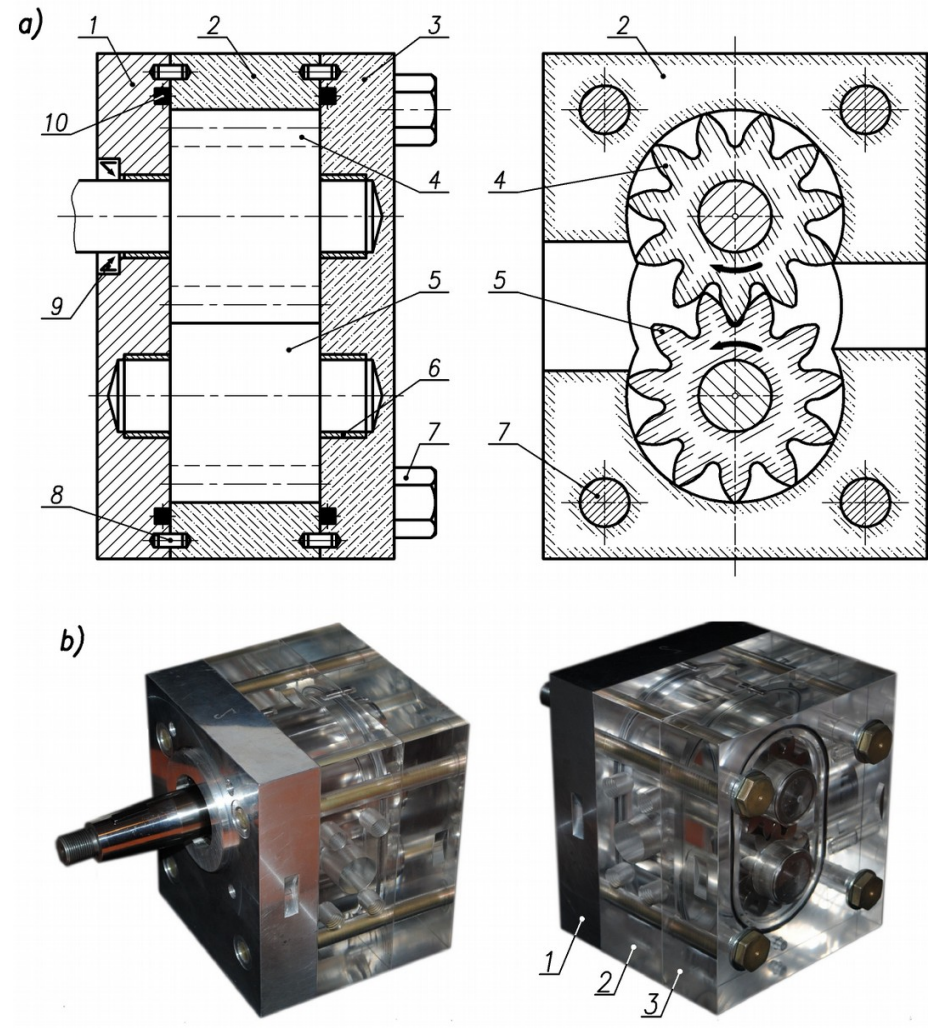

Fig. 3. Experimental gear pump

a) pump design diagram, b) pump view

The pump body consists of three parts: the front (1), the middle (2) and the back (3). The body parts are positioned by pins (8) and bolted (7). In the body, there is a set of two gears featuring shafts: the active gear (4) and the passive gear (5). The shafts of the gears rotate in 
the hydrostatic slide bearings (6). The drive shaft of the pump is sealed with a Simmer ring (9), whereas the bodies are sealed with O-rings (10).

The actual view of the pump is shown in Fig. 3b. The front body (1) is made of an aluminium alloy, while the middle (2) and the back (3) are made of technical glass (PMMA), which allows for the observation of the flow phenomena and processes inside the pump. The need for the application of the transparent technical glass featuring low mechanical properties, results in the visualization study being carried out only at low and medium working pressure values.

\subsection{Ultrasonic emitter}

Fig. 4a shows the ultrasonic emitter designed by a team of researchers from National Technical University of Ukraine.
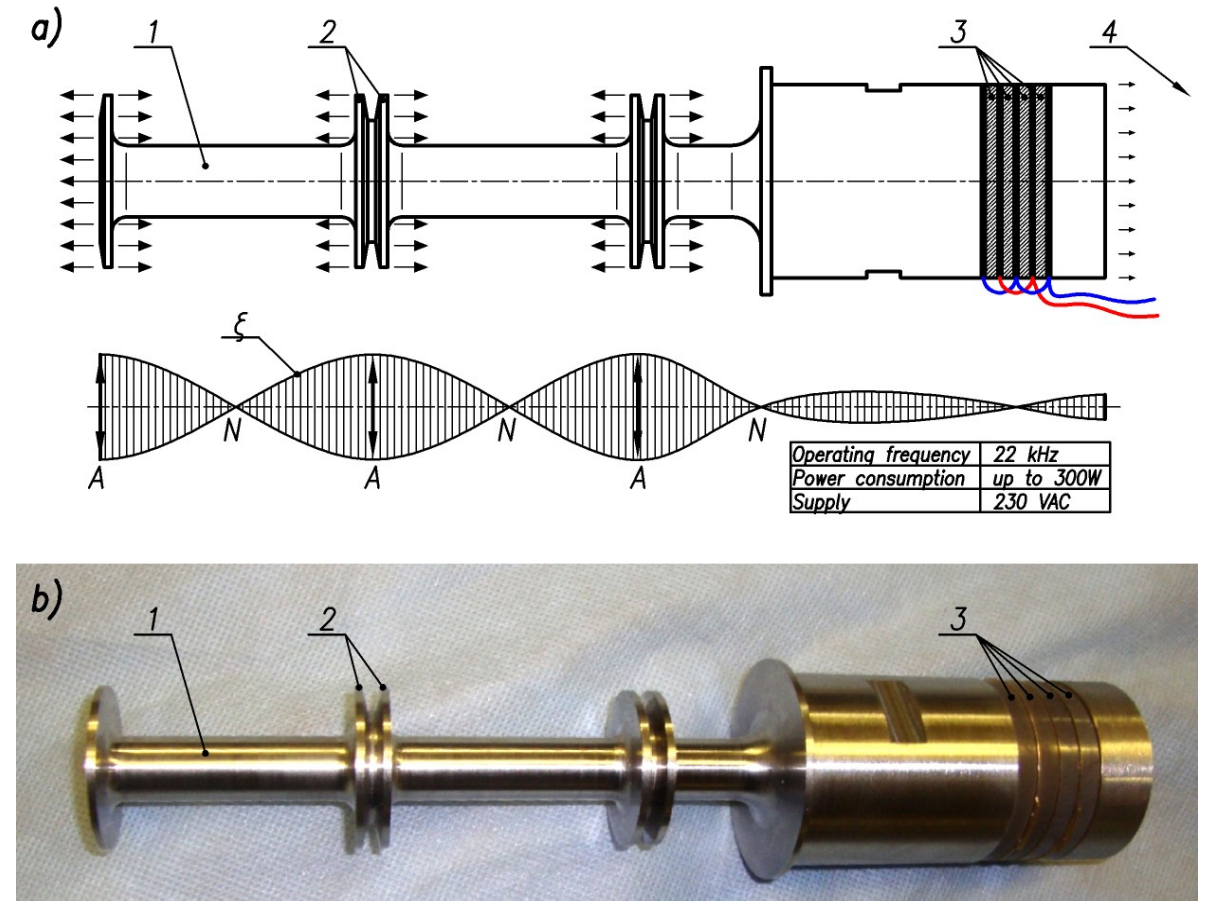

Fig. 4. Ultrasonic resonant drive-emitter with a developed radiation surface a) design diagram, b) actual view

The ultrasonic emitter consist of a custom shaped ultrasonic emitter (1) featured radiating disks (2), placed in the anti-nodes (A) of a standing ultrasonic wave ( $\xi$ ), and a piezoelectric transducer (3) which is the causative agent of vibration oscillations. The ultrasonic emitter, mounted vertically in a hydraulic tank and immersed in a working fluid, is excited by a custom designed, electronic oscillator (4).

The actual view of the ultrasonic emitter is shown in Fig. $4 \mathrm{~b}$.

\section{Experimental research}

Experimental research was carried out for the following parameter values:

- rotational speed of the pump

- inlet pressure of the pump

$500 \pm 10 \mathrm{rev} / \mathrm{min}$,

$0 \pm 0.05$ bar, 
- outlet pressure of the pump

- temperature of the working fluid

- ultrasonic emitter power
$10 \pm 0.1$ bar,

$21 \pm 2^{\circ} \mathrm{C}$,

$300 \mathrm{~W}$.

The research was carried out in 4 stages. At the first stage, the visualization research was carried out for hydraulic oil which was not subject to a degassing process. Then, the degassing system was started for 30 minutes at full power, after which the visualization research was carried out again. This cycle was repeated twice, which allowed to determine the effect of the degassing system working for a total of 90 minutes.

The nomenclature used in the article on the forms and intensity of cavitation phenomena is described in detail in [10].

The research results, for two exemplary gear set positions, are shown in Fig. 5.
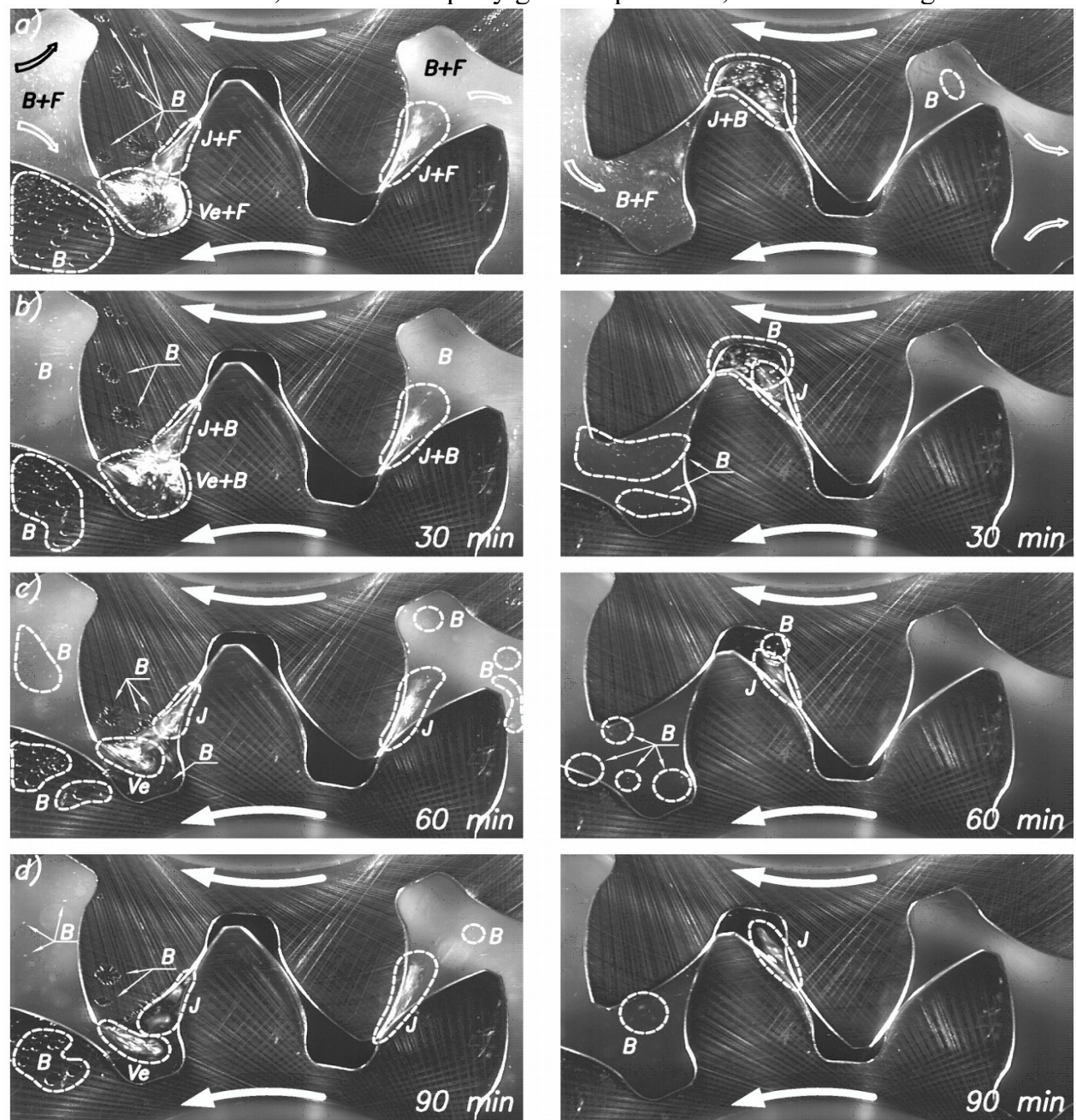

Fig. 5. The real flows in the outlet bridge zone for different degassing times

It is perfectly evident that in the case of the non-degassed oil (Fig. 5a), there is a number of areas in the pump, where cavitation phenomena are clearly visible. What is more, the intensity of those phenomena is high, as evidenced by the occurrence of cavitation foam $(\mathrm{F})$, cavitation bubble (B) or both $(\mathrm{B}+\mathrm{F})$. These phenomena are so intense that cavitation bubbles occur even in the axial gap between the surface of the body and the surface of the gear. In addition to bubbles (B) and cavitation foam (F), one can also notice two other 
forms of cavitation phenomena, namely veil (Ve), occurring near the tooth's head, and jet (J) appearing in the inter-tooth gap. However, also in that case, those phenomena are accompanied by a cavitation foam $(\mathrm{J}+\mathrm{F}, \mathrm{Ve}+\mathrm{F})$ or cavitation bubbles $(\mathrm{J}+\mathrm{B})$.

Tests performed after 30 minutes of the degassing system operation (Fig. 5b) showed that the intensity of the cavitation phenomena decreased. Evidence of that was the disappearance of cavitation foam (F) and the limitation of the cavitation bubbles (B) occurrence area.

After 60 minutes of the degassing system operation (Fig. 5c), it was noticed that the intensity of cavitation phenomena was still decreasing, and some additional forms of cavitation, namely veil (V) and jet (J), were already alone.

Finally, after 90 minutes of the degassing system operation (Fig. 5d), it was concluded that virtually all cavitation bubbles (B) had been eliminated, and inside the pump only other forms of cavitation phenomena were visible, namely veil (Ve) and jet (J).

\section{Conclusions}

The presented research has proved that the ultrasonic degassing system works as expected and has a significant influence on the gas content in a hydraulic oil as well as on a hydraulic gear pump operation. Besides, the study has clearly shown that the reduction in the amount of gas dissolved in the medium causes a radical decrease of the cavitation embryos and, due to that, reduction of the cavitation phenomenon practically in all characteristic areas of the pump. Finally, that results in the noticeable reduction of noise and improvement of the hydraulic characteristics as well as overall performance of the pump.

\section{References}

[1] А. Луговской, Н. Чухраев, Видавничо-поліграфічний центр «Київський університет», p. 244 (2007) (in Ukrainian).

[2] А. Перни, Судостроение, p. 438 (1988) (in Ukrainian).

[3] И. Голямина (ред.), Маленькая энциклопедия, p. 400 (1979) (in Ukrainian).

[4] О. Луговський, А. Зілінський, І. Гришко, Вісник НТУУ «КПІ». Машинобудування, Вип. 74 (2015), p. 11-17. (in Ukrainian).

[5] P. Antoniak, 7th IFK, Vol. 2 (2010), p. 501-510.

[6] P. Antoniak, 6th FPNI-PhD Symposium, Vol. 2 (2010), p. 691-698.

[7] J. Stryczek, P. Antoniak., O. Jakhno, D. Kostuk, A. Kryuchkov, G. Belov, L. Rodionov, Archives of Civil and Mechanical Engineering, Vol.15, Issue 1 (2015), p. 95-108. https://doi.org/10.1016/j.acme.2014.02.010.

[8] V. Sahoo, U. Mukherjee, M. Das, R. Maiti, P. Antoniak, J. Stryczek, L. Rodionov, Journal of Flow Visualization and Image Processing, Vol. 23 (2016), p. 345-376.

[9] J. Stryczek, Z. Lurye, V. Solovyov, P. Antoniak, Bulletin of National Technical University "KhPI", nr 42 (2017), p. 7-13.

[10]P. Antoniak, J. Stryczek, Archives of Civil and Mechanical Engineerin, vol. 18 (2018), p. 1103-1115. https://doi.org/10.1016/j.acme.2014.02.010 